\title{
Tips for oral medicine in primary care
}

\author{
Mike Lewis ${ }^{1}$ continues our new series of columns providing 'top tips' in dental specialties.
}

$\mathrm{O}$ ral medicine is the specialty of dentistry concerned with the oral healthcare of patients with chronic, recurrent and medically related disorders of the oral and maxillofacial region and with their diagnosis and non-surgical management. As such, oral medicine sits at the interface between dentistry and medicine. An effective collaboration between the dentist and the general medical practitioner (GMP) will permit many patients with oral medicine problems to be successfully managed in primary care without the need for referral to secondary care. Such multiprofessional working is of direct benefit to the patient and reduces pressure on specialist services, which are then able to deal with the more complex cases. The aim of this short article is to present some 'clinical tips' that will help dental primary care practitioners manage oral medicine conditions.

1. Assessment of the orofacial tissues. An abnormality in the orofacial tissues is likely to be initially detected visually. However, manual palpation may also reveal a change. As a basic principle, a sinister condition, such as a carcinoma, is firm to palpation (indurated) whilst a non-sinister abnormality, for example cyst, is soft to palpation. All visual and palpable findings should be recorded in the clinical notes. The use of the term 'lesion', which is derived from the Latin noun 'laesio' meaning injury, should be avoided since in a descriptive sense it is effectively meaningless. It is preferable to refer to a change within the orofacial tissue as an 'abnormality' and use descriptive terms such as ulcer, red patch or swelling. ${ }^{1}$ This is particularly relevant in the written text of a patient referral since it may influence the degree of urgency during vetting by secondary care. In addition, the usefulness of clinical photography cannot be over-emphasised both for the monitoring of an abnormality in the practice or for inclusion of an image within a referral.

2. Special investigations. The range of special investigations available to assist diagnosis and monitoring of patients with orofacial disease is extensive. Liaison with the medical practitioner to obtain the results of basic tests is really helpful. For oral mucosal disease, the most useful haematological investigations are full blood count (FBC), folate, vitamin $\mathrm{B}_{12}$ and ferritin. HbAlc is the most reliable indicator of diabetic status in relation to a patient with dry mouth or oral candidosis.

3. Prescribing. The British National Formulary (BNF), which includes the Dental Practitioner Formulary (DPF), provides the most comprehensive source of advice on all aspects of prescribing including interactions and adverse drug reactions. ${ }^{2}$ However, the Scottish Dental Clinical Effectiveness Programme (SDCEP) guidelines for dental prescribing provide concise information in the surgery. ${ }^{3}$ The use of abbreviations, such as t.d.s or t.i.d, for frequency of dosing should be avoided both in prescription writing and case records. It is preferable to write 'every 8 hours' or 'three times daily' to avoid any potential misinterpretation.
4. Management of pain. Orofacial pain of non-dental origin is not usually associated with visible clinical signs and therefore diagnosis needs to be based on a detailed assessment of the symptoms. ${ }^{4}$ Severity of the pain should be recorded on a scale of zero to ten, where 0 corresponds to 'no pain' and 10 indicates 'the worst pain ever experienced'. The patient should be asked to describe the nature of the pain. Do not suggest particular words but ask the question, 'How would you describe the nature of the pain?' The response often involves either 'shooting, 'burning' or 'gripping'. As a generalisation, a ' 10 out of 10' shooting pain that lasts seconds is trigeminal neuralgia (any score of less than 10 would suggest that it is not trigeminal neuralgia) whilst an ' 8 out of 10 ' burning sensation that lasts all day is likely to be burning mouth syndrome (BMS). A ' 7 out of 10 ' gripping ache that is constant is probably persistent idiopathic facial pain (PPIF).

a. Trigeminal neuralgia: This is the worst pain that the patient will have ever suffered (women often reporting it to be worse than childbirth) so it is essential to provide relief as soon as possible. Carbamazepine (in DPF) should be prescribed as a $100 \mathrm{mg}$ tablet twice daily for ten days. The GMP should be informed along with a request for a baseline $\mathrm{FBC}$ and liver function tests. If trigeminal neuralgia is present, then provision of carbamazepine will result in some improvement in symptoms within 48 hours, a finding that helps confirm the diagnosis.

b. Burning mouth syndrome: The oral mucosa will appear normal in burning mouth syndrome (BMS). Patients with BMS are characteristically anxious about the presence of sinister disease within the mouth, in particular cancer (known as cancerphobia). The patient will never ask 'Do you think I have cancer?' However, it is essential to raise this issue and provide strong reassurance that there is no evidence of sinister disease. The patient should also be reassured that BMS is a common condition within the population. Interestingly, sufferers have never heard of the condition or met anyone else who may have it. There will be a need for GMP liaision to exclude other aetiological systemic factors and discuss the likely use of antidepressant medication (usually low-dose amitriptyline at night) for 3-6 months.

c. Persistent idiopathic facial pain: If a dental cause for the orofacial pain has been excluded and PIFP is suspected, then liaison with the GMP to consider provision of a low dose of an antidepressant drug, such as amitriptyline, at night is appropriate.

4. Dry mouth (xerostomia): Apart from an obvious lack of saliva pooling in the floor of the mouth at rest, a simple test to determine if a patient has a dry mouth is to place an examination mirror against the buccal mucosa. If the face of the mirror sticks, then it is likely that there is reduced or altered saliva production. The most frequent cause of dry mouth is an adverse event associated with drug therapy so the potential for alternative medication needs 
4

to be considered with the GMP. It is also essential to exclude diabetes by requesting an $\mathrm{HbAlc}$ test from the GMP.

5. Mucosal disorders.

a. Recurrent aphthous stomatitis (RAS): For frequent sufferers, it is useful to request results of basic haematological tests (see above). If present, the underlying cause of any haematinic deficiency (most frequently iron) needs to be identified and corrected by the GMP. All patients with RAS should be given dietary advice on the avoidance of tomatoes and benzoate-based preservatives (E210-219), in particular carbonated soft drinks, since hypersensitivity to these foodstuffs may be involved.

b. Lichen planus: The characteristic presentation of lichen planus involves a bilateral and symmetrical distribution of lacy white lines. An asymmetrical distribution or a physical contact with an amalgam restoration is likely to be a lichenoid reaction. If the lateral margin of the tongue is involved, it is essential to refer for a biopsy to exclude epithelial dysplasia or carcinoma.

c. Geographic tongue: Patients with geographic tongue are often anxious that the mucosal changes represent an infection or malignancy. Reassurance that it is neither of these conditions is essential. Symptomatic geographic tongue will often respond to a zinc sulphate mouthwash (not in DPF). Liaise with GMP to prescribe effervescent zinc sulphate $125 \mathrm{mg}$ dissolved in $10 \mathrm{ml}$ and used as a mouthwash for two minutes three times daily. This preparation is also helpful in patients with a complaint of altered or metallic taste.

d. Candidosis: The basic principle to the management of any form of oral candidosis, historically referred to as the 'disease of the diseased', is identification of the underlying predisposing factor which is promoting the opportunistic infection. ${ }^{5} \mathrm{~A}$ wide range of local and medical factors have to be investigated, some requiring the results of routine haematological tests from the GMP (see above). The agent of choice for the treatment of the majority of cases of oral candidosis is fluconazole (50 mg capsule once daily for seven days). Topical antifungals have limited benefit.

e. Red and white patches: The vast majority of cases of leukoplakia will never undergo malignant transformation. However, a red patch (erythroplakia) or a red/white patch (erythroleukoplakia) are both associated with a high risk of cancer and as such should be referred, including a photograph, as urgent suspected cancer (USC) according to NICE guidelines.

\section{References}

1. NICE. British National Formulary. Available at: https://bnf.nice.org.uk/ (accessed December 2021).

2. Scottish Dental Clinical Effectiveness Programme Drug prescribing for dentistry. 3rd edition. Last updated June 2021. Available at: https://www.sdcep.org.uk/publishedguidance/drug-prescribing/ (accessed December 2021).

3. Lewis M. Mouth cancer - What's it to you? Br Dent J 2018; 225: 789-790.

4. Lewis M A 0, Lamey P-J. Orofacial pain. In Oral medicine in primary dental care. Switzerland: Springer Nature, 2019.

5. Lewis M, Williams D. Diagnosis and management of oral candidosis. Br Dent J 2017; 223: 675-681.

\section{Invitation to contribute}

If you are a dental specialist, could you compile a list of your top tips in that dental specialty for $B D J$ readers? If yes, please contact News Editor Kate Quinlan, k.quinlan@nature.com.

\section{How good is your oral medicine?}

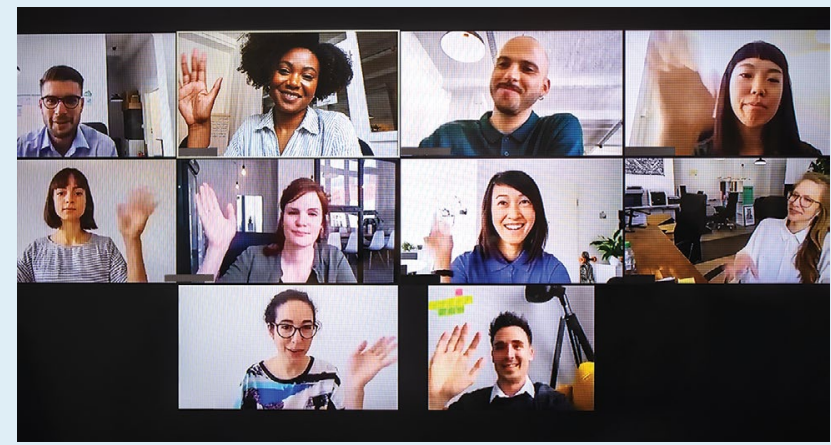

The British Dental Association (BDA) will be hosting a webinar on Thursday 20 January 2022 from 19:30-20:30 on 'How good is your oral medicine?', featuring Professor Mike Lewis as speaker.

This webinar will provide the participant with an understanding of how to diagnose and provide initial management of those orofacial conditions that present most frequently in dental primary care, and will include one hour of CPD.

The event is free for BDA members. To book your place please visit www.bda.org/ilearn. 